www.nature.com/ja

\title{
Immunomodulatory effects of azithromycin on serum amyloid A production in lipopolysaccharide-induced endotoxemia in mice
}

\author{
Vanesa Ivetić Tkalčević, Boška Hrvačić, Ivanka Pašalić, Vesna Eraković Haber and Ines Glojnarić
}

The Journal of Antibiotics (2011) 64, 515-517; doi:10.1038/ja.2011.14; published online 18 May 2011

Keywords: azithromycin anti-inflammatory activity; azithromycin immunomodulation; endotoxemia; IL-6; LPS; serum amyloid A

Growing number of scientific investigations indicate that certain macrolides, a well-known group of antibiotics isolated from actinomycetes, also exert anti-inflammatory and immunomodulatory properties by modulating leukocyte function and circulating concentrations of proinflammatory cytokines. These macrolide properties have been employed in various chronic inflammatory disorders, such as diffuse panbronchiolitis, chronic obstructive pulmonary disease and cystic fibrosis. ${ }^{1,2}$

Recently, azithromycin (AZI), clarithromycin and telithromycin were shown to inhibit acute inflammatory processes in a murine model of lipopolysaccharide (LPS)-induced sepsis. ${ }^{3-5}$ The pathogenesis of sepsis involves a progressive and dynamic expansion of the systemic inflammatory response to bacterial infection. ${ }^{6} \mathrm{~A}$ major factor contributing to sepsis is the shedding of LPS from the cell wall of Gram-negative bacteria into the circulation. In response to infection and inflammatory stimuli, hepatocytes produce serum amyloid A (SAA) protein, which represents a family of low-molecular-weight acute-phase proteins. ${ }^{7}$ The main stimuli to the synthesis of SAA by the liver are cytokines, such as interleukin (IL)-6, IL-1 $\beta$ and tumor necrosis factor- $\alpha .^{8}$

As our previously published report showed that anti-inflammatory activity of AZI inhibits LPS-induced plasma tumor necrosis factor- $\alpha$ production and neutrophil infiltration in lungs, and prolongs survival of experimental animals, ${ }^{3}$ we aimed this study to further elucidate AZI anti-inflammatory activity on LPS-induced SAA secretion, plasma IL-6 production and its effects on blood cell count.

\section{EXPERIMENTAL PROCEDURE}

\section{Animals}

Male BALB/cJ mice 8- to 10-week old were obtained from Charles River (Lyon, France). Eight animals per group were kept on wire mesh floors with irradiated maize granulate bedding (Scobis Due, Mucedola, Italy) and maintained under standard laboratory conditions (temperature $23-24^{\circ} \mathrm{C}$, relative humidity $60 \pm 5 \%, \sim 15$ air changes per hour and artificial lighting with circadian cycle of $12 \mathrm{~h}$ ). Food (Standard food for mice and rats, 4R21, Mucedola, Milan, Italy) and tap water were provided ad libitum. Mice were allowed to acclimatize for 10 days before the beginning of each experiment.

All procedures on animals were performed in accordance with (1) the EEC Council Directive 86/609 of 24 November 1986 on the approximation of laws, regulations and administrative provisions of the Member States regarding the protection of animals used for experimental and other scientific purposes; and (2) Statute of Republic Croatia, Animal Welfare Law, NN 135 of 13 December 2006.

\section{LPS-induced endotoxemia}

Experimental model of LPS-induced endotoxemia was conducted, as previously described. ${ }^{3}$ Briefly, $25 \mu \mathrm{g}$ of LPS from Escherichia coli (serotype 0111:B4; Sigma, St Louis, MO, USA) in $0.2 \mathrm{ml}$ of sterile saline was administered i.p. to mice, except those in the healthy control group that were treated with the same volume of sterile saline. AZI (200 $\mathrm{mg} \mathrm{kg}^{-1}$; PLIVA d.d., Zagreb, Croatia) and vehicle $(5 \%(\mathrm{v} / \mathrm{v})$ dimethyl sulfoxide (Sigma) and $0.5 \%(\mathrm{w} / \mathrm{v})$ methylcellulose (Sigma)) were administered orally by gavage $30 \mathrm{~min}$ before injection of LPS. At indicated time points, blood was collected by puncturing the vena jugularis and arteria carotis communis for future analysis of white blood cell (WBC), neutrophil, lymphocyte and monocyte counts, and for the analysis of IL-6 and SAA concentrations.

Subsequently, healthy animals were treated orally with AZI $\left(200 \mathrm{mg} \mathrm{kg}^{-1}\right)$ or vehicle. At indicated time points, blood was collected, as previously described, for future analysis of serum SAA concentration.

\section{Hematological and clinical biochemical variables}

Whole blood was analyzed for WBC and differential leukocyte count on a Sysmex SF 3000 hematological analyser (Sunrise Co Ltd, Kobe, Japan).

To obtain plasma/serum for biochemical tests, blood was collected in tubes with plasma/serum separator and centrifuged for $15 \mathrm{~min}$ at $2000 \times \mathrm{g}$. Plasma/ serum was transferred into new tubes and frozen at $-20^{\circ} \mathrm{C}$ for further IL- 6 and SAA analysis. 
To determine the concentration of IL- 6 and SAA, mouse enzyme-linked immunosorbent assay was performed using capture and detection antibodies according to the manufacturer's recommendations (IL-6, R\&D Systems, Minneapolis, MN, USA; SAA, Tridelta, County Kildare, Ireland).

\section{Data analysis and statistical evaluation}

Differences in observed values were analyzed statistically using the MannWhitney test. All statistical analyses were done using the program GraphPad InStat v. 4.0 (La Jolla, CA, USA). The level of significance was set at $P<0.05$ in all cases.

The i.p. LPS administration markedly decreased WBC number in the first $3 \mathrm{~h}$ after application (Figure 1a). Differential cell counts revealed decreased neutrophil and lymphocyte counts (Figures $1 \mathrm{~b}$ and c). However, starting at $5 \mathrm{~h}$ after inflammation induction, number of neutrophils markedly increased (Figure $1 \mathrm{~b}$ ). Oral treatment with AZI at $200 \mathrm{mg} \mathrm{kg}^{-1}$ prevented reduction of WBC number at 0.25 and $1.5 \mathrm{~h}$ after LPS administration, which accounted for prevented decrease of lymphocyte count $(0.25 \mathrm{~h})$ and neutrophil count $(1.5 \mathrm{~h}$; Figures 1a-c). Also, AZI significantly reduced neutrophil number $24 \mathrm{~h}$ after LPS-induced inflammation (Figure 1b). There was no significant effect of AZI on LPS-induced reduction of monocyte number (data not shown).

Orally administered AZI (200 mg kg-1) induced significant increase in SAA concentration 3-5 h after LPS administration (Figure 2a). LPS significantly increased serum SAA concentration $24 \mathrm{~h}$ after administration (Figure 2a). At that time point, AZI significantly decreased the effect of LPS on SAA (Figure 2a).

Administered orally ( $200 \mathrm{mg} \mathrm{kg}^{-1}$ ) to healthy animals, AZI induced significant increase in serum SAA concentration at abserved time points (Figure $2 \mathrm{~b}$ ).

Systemic application of LPS provoked significant sustained increase in plasma concentration of IL-6, the stimuli to SAA production, $1.5-5 \mathrm{~h}$ after LPS administration (Figure 2c). Orally administered, AZI $\left(200 \mathrm{mg} \mathrm{kg}^{-1}\right)$ markedly increased IL-6 concentration $3 \mathrm{~h}$ after the induction of endotoxemia (Figure 2c).

In the experimental model of LPS-induced endotoxemia, we have shown that systemically administered LPS induces transient reduction in WBC count and sustained increase in plasma IL- 6 concentration, as well as increase in serum SAA concentration and neutrophil number in plasma in the later phase of the inflammatory reaction. This finding partially confirms a previous study performed by Copeland et al., ${ }^{9}$ which showed that, in blood, LPS triggers the secretion of several proinflammatory cytokines, such as tumor necrosis factor- $\alpha$, IL- 6 and IL-1, and induces transient lymphopenia, vasodilatation, impaired coagulation and fibrinolysis.

AZI anti-inflammatory activity on blood cell count, after oral administration at $200 \mathrm{mg} \mathrm{kg}^{-1}$, is in accordance with our previously published report indicating that AZI inhibits acute inflammatory processes involved in LPS-induced endotoxemia. ${ }^{3}$ However, besides anti-inflammatory activity, AZI exerted the immunomodulatory effects as well. In the first $3 \mathrm{~h}$ after inflammation induction, as well as in healthy animals, AZI significantly enhanced SAA production, whereas in the later stages of LPS-induced inflammatory reaction, AZI ameliorated the increase in SAA concentration. Bone ${ }^{10}$ reported that the extreme changes in the concentration of acute-phase proteins induced by overemphasized cytokine activity can result in death, a possible outcome of septic shock. As SAA is synthesized by hepatocytes in response to infection and inflammatory stimuli, its concentration is a valuable indicator in monitoring the efficacy of antimicrobial and anti-inflammatory therapy. ${ }^{8}$
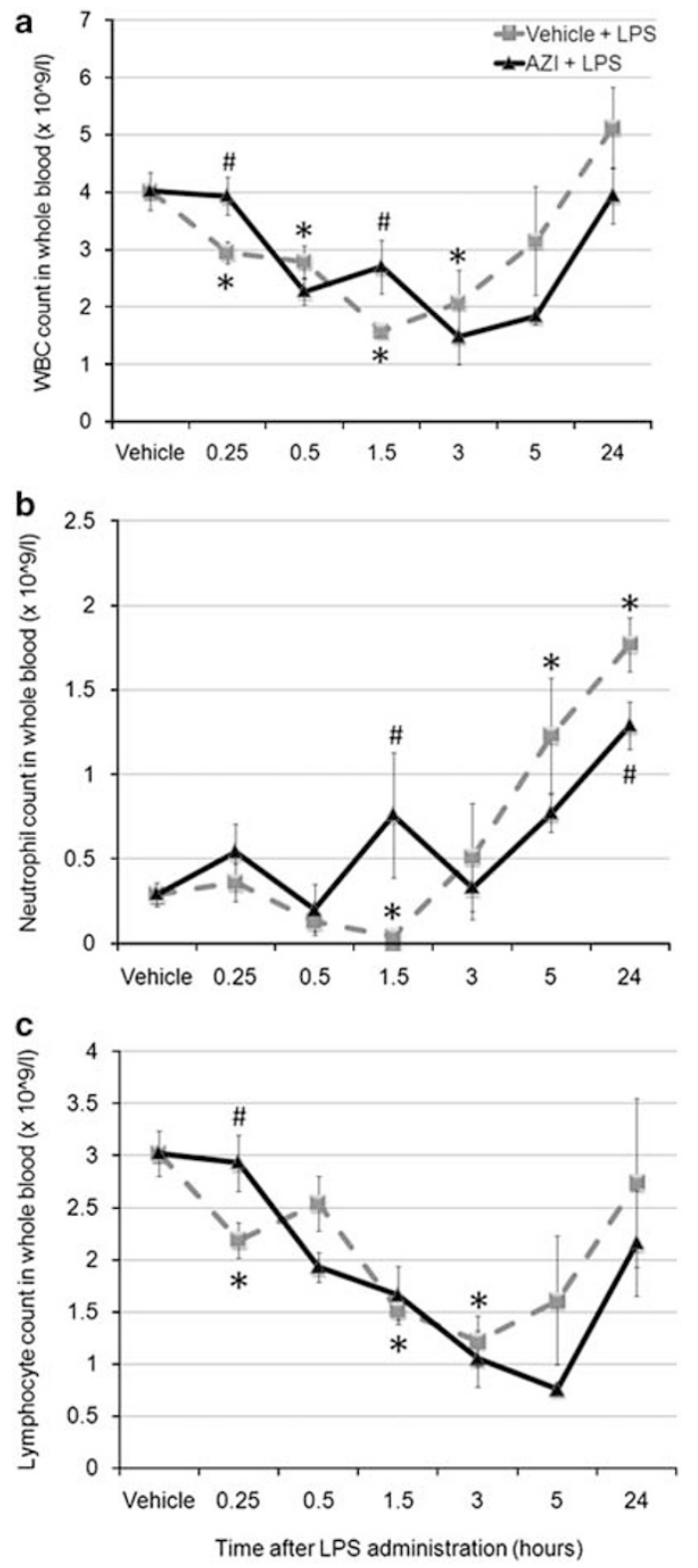

Figure 1 Effects of orally administered azithromycin $\left(200 \mathrm{mg} \mathrm{kg}^{-1}\right)$ on white blood cell count (a), as well as neutrophil (b) and lymphocyte (c) number at indicated time points after i.p. lipopolysaccharide (LPS; $25 \mu \mathrm{g}$ per mouse) administration in mice. Data are presented as group average \pm s.e.m. Sign * indicates difference from vehicle-treated healthy animals and sign \# from vehicle- and LPS-treated animals at the same time point; $P<0.05, n=8$.

Published data indicate that macrolides show immunomodulatory activity by influencing cytokine production by several cell types and by altering polymorphonuclear cell functions. ${ }^{11}$ Also, Gorrini et al. ${ }^{12}$ showed that in contrast to long-term treatment, which inhibits inflammatory reactions, short-term treatment with roxithromycin provokes an immunological response. This finding could be beneficial in the treatment of sepsis, which has extremely short pathogenesis. We have already indicated that AZI and possibly other macrolides with similar anti-inflammatory properties could be considered as potential adjuvant anti-inflammatory therapeutic agents in the treatment of sepsis by inhibiting the initial tumor necrosis factor- $\alpha$ response. ${ }^{3}$ The findings presented here further demonstrate the ability of single oral 

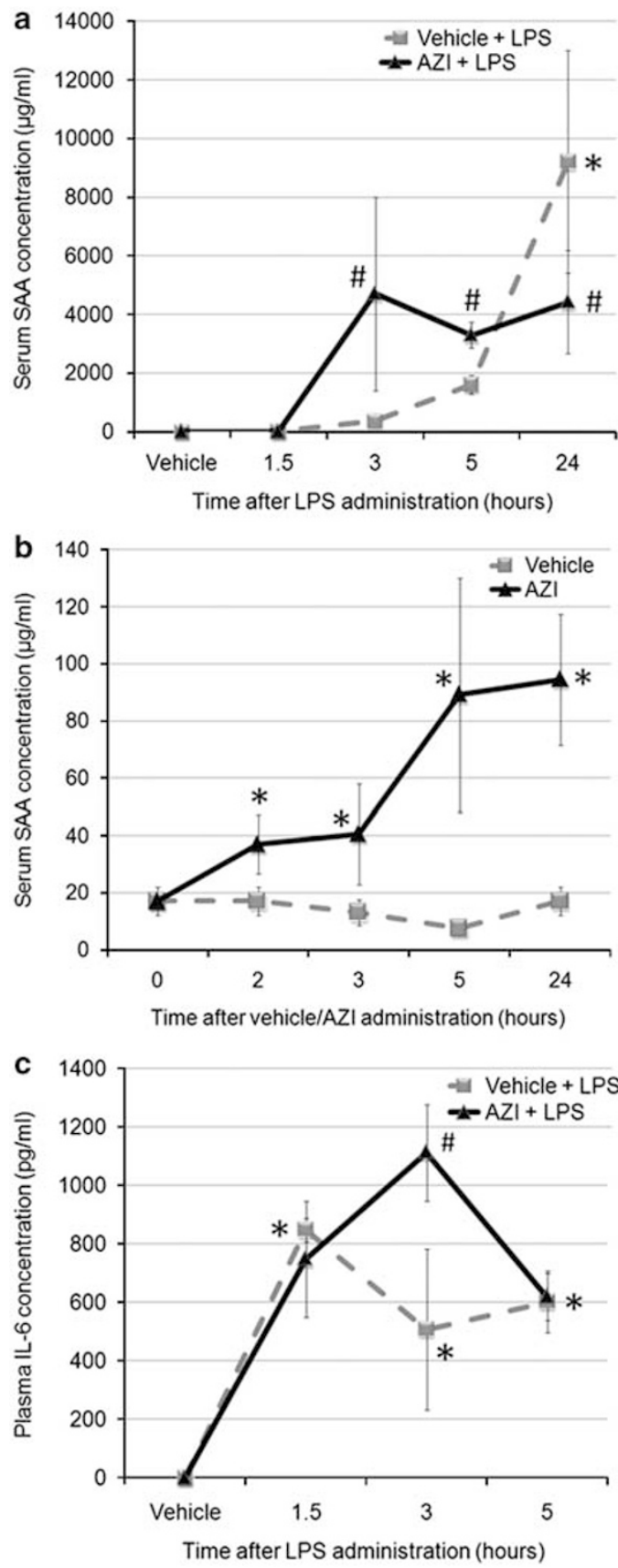

Figure 2 Effects of orally administered azithromycin (AZI; $200 \mathrm{mg} \mathrm{kg}^{-1}$ ) on (a) serum amyloid $A(S A A)$ concentration at indicated time points after i.p. lipopolysaccharide (LPS; $25 \mu \mathrm{g}$ per mouse) administration, (b) SAA concentration at indicated time points after AZI/vehicle administration and (c) plasma interleukin- 6 concentration at indicated time points after LPS administration in mice. Data are presented as group average \pm s.e.m. Sign * indicates difference from appropriate vehicle-treated healthy animals and sign \# from vehicle- and LPS-treated animals at the same time point; $P<0.05, n=8$.

AZI dose to stimulate the initial immune response and to inhibit later enhanced inflammatory reaction.

As IL-6 is considered as one of the stimuli of SAA synthesis, ${ }^{8}$ the effects of AZI on LPS-increased plasma IL-6 concentration were investigated. AZI significantly increased LPS-induced IL-6 synthesis, and at the same time, it increased SAA concentration. Cytokine IL-6 is generally regarded as an inflammation-modulating cytokine. ${ }^{2}$ In human monocytes, both stimulatory and inhibitory effects of some macrolide antibiotics have been observed on IL-6. ${ }^{13,14}$ Therefore, it is possible that by increasing plasma IL- 6 concentration, AZI induced SAA secretion.

In conclusion, we have defined more precisely the immunomodulatory activity of AZI in LPS-induced endotoxemia. AZI exerted antiinflammatory activity by preventing the initial neutropenia and lymphopenia and in the later stage of the inflammatory reaction by inhibiting LPS-induced increase in blood neutrophil number. In healthy animals, AZI increased the concentration of serum SAA. The same was observed at the induction phase of the inflammatory reaction when AZI increased LPS-stimulated SAA synthesis, possibly by induction of plasma IL- 6 secretion. In the later stage of inflammation, AZI ameliorated LPS-induced increase in serum SAA concentration. Results presented indicate that AZI, administered at the beginning of LPS-induced endotoxemia, acts as a proinflammatory signal. On the other hand, it tends to lessen the longer-term severe increases in inflammatory mediators induced by LPS. These actions of AZI may provide additional therapeutic benefit in sepsis and also suggest potential new directions for inhibitors of inflammatory reactions in sepsis.

\section{ACKNOWLEDGEMENTS}

This work was supported by GlaxoSmithKline Research Centre Zagreb. We thank Ms S Skender, M Horvatinčić and R Povrženić, and Mr H Poduška and V Vrban for their excellent technical assistance.

1 Amsden, G. W. Anti-inflammatory effects of macrolides-an underappreciated benefit in the treatment of the community acquired respiratory tract infections and chronic inflammatory pulmonary conditions? J. Antimicrob. Chemother. 55, 10-21 (2005).

2 Čulić, O., Eraković, V. \& Parnham, M. J. Anti-inflammatory effects of macrolide antibiotics. Eur. J. Pharmacol. 429, 209-229 (2001).

3 Ivetić Tkalčević, V. et al. Anti-inflammatory activity of azithromycin attenuates the effects of lipopolysaccharide administration in mice. Eur. J. Pharmacol. 539, 131-138 (2006).

4 Ivetić Tkalčević, V. et al. The anti-inflammatory activity of clarithromycin inhibits TNF $\alpha$ production and prolongs survival following lipopolysaccharide administration in mice. Int. J. Antimicrob. Agents 32, 195-196 (2008).

5 Leiva, M., Ruiz-Bravo, A., Moreno, E. \& Jimenez-Valera, M. The anti-inflammatory activity of telithromycin in a mouse model of septic shock. Int. J. Antimicrob. Agents 29, 364-365 (2007).

6 Shapiro, L. \& Gelfand, J. A. Cytokines and sepsis: pathophysiology and therapy. N. Horiz. 1, 13-22 (1993).

7 Gabay, C. \& Kushner, I. Acute-phase proteins and other systemic responses to inflammation. N. Engl. J. Med. 340, 448-454 (1999).

8 Uhlar, C. M. \& Whitehead, A. S. Serum amyloid A, the major vertebrate acute-phase reactant. Eur. J. Biochem. 265, 501-523 (1999).

9 Copeland, S., Warren, H. S., Lowry, S. F., Calvano, S. E. \& Remick, D. Acute inflammatory response to endotoxin in mice and humans. Clin. Diagn. Lab. Immunol. 12, 60-67 (2005)

10 Bone, R. C. Toward a theory regarding the pathogenesis of the systemic inflammatory response syndrome: what we do and do not know about cytokine regulation. Crit. Care Med. 24, 163-172 (1996).

11 Schultz, M. J. Macrolide activities beyond their antimicrobial effects: macrolides in diffuse panbronchiolitis and cystic fibrosis. J. Antimicrob. Chemother. 54, 21-28 (2004).

12 Gorrini, M. et al. Inhibition of human neutrophil elastase by erythromycin and flurythromycin, two macrolide antibiotics. Am. J. Respir. Cell. Mol. Biol. 25, 492-499 (2001).

13 Bailly, S., Pocidalo, J. J., Fay, M. \& Gougerot-Poicidalo, M. A. Differential modulation of cytokine production by macrolides: interleukin- 6 production is increased by spiramycin and erythromycin. Antimicrob. Agents Chemother. 35, 2016-2019 (1991).

14 Khan, A. A., Silfer, T. R., Araujo, G. \& Remington, J. S. Effects of clarithromycin and azithromycin on production of cytokines by human monocytes. Int. J. Antimicrob. Agents 11, 121-132 (1999). 